\title{
Dexterous Cooperative Manipulation with Redundant Robot Arms
}

\author{
David Navarro-Alarcon, Vicente Parra-Vega, \\ Silvionel Vite-Medecigo, and Ernesto Olguin-Diaz \\ Grupo de Robótica y Manufactura Avanzada, CINVESTAV-Saltillo, México \\ \{david.navarro, vicente.parra, silvionel.vite\}@cinvestav.edu.mx
}

\begin{abstract}
A novel model-based force-position control scheme for cooperative manipulation tasks with redundant arms is proposed in this paper. Employing an orthogonal decomposition of the object contact mechanics, independent pose and force trajectory tracking can be achieved. In this way, a high precision cooperative scheme is enforced since the projection of the object velocity into the contact normal direction converges to zero, improving the system cooperativeness. Simulation results are presented for a humanoid torso to visualize its closed-loop performance.
\end{abstract}

\section{Introduction}

Multi-arm cooperative schemes have been a topic of special interest for the last 20 years. This comes from the fact that multiple arms working in a given task can improve the execution dexterity, increase the pay load capability, provide higher manipulation flexibility, among other advantages [1]. Moreover, multiarm cooperative schemes can be employed to model certain robot manipulation tasks such the robotic hand wherein each finger is modeled as an arm, and the humanoid robot manipulation where two anthropomorphic arms manipulate an object. Notice that these manipulation tasks involve physical interaction among the cooperators, consequently, contact forces arise when manipulating an object. Then, in order to provide a stable physical interaction, the object position trajectory and the exerted contact force must be simultaneously controlled among the participants. It must be remarked that these two problems are not fully decoupled as the case of fixed holonomic constraints 2]. Therefore, in cooperative schemes the object is now allowed to move in the same direction as the exerted contact force.

On the other hand, when redundant robot arms, like the humanoid anthropomorphic arms, are employed for cooperative tasks the benefits and possibilities are increased even more. This as a result of the multiple kinematic configurations available for a given end-effector pose (position+orientation). Even more, with the use of redundancy a cooperative system can avoid collisions with obstacles while manipulating the object, reconfigure itself in order to optimize the power consumption while exerting a given contact force, or just to optimize a meaningful cost function. In this sense, redundancy can help to improve the dexterity of the robotic system.

E. Bayro-Corrochano and J.-O. Eklundh (Eds.): CIARP 2009, LNCS 5856, pp. 910 917, 2009.

(C) Springer-Verlag Berlin Heidelberg 2009 
The main contribution of this paper is the enforced stability in the Lyapunov sense of the normal velocity-position error manifold, presented here as cooperativeness error. Therefore, the stable execution of independent force and position tracking is guaranteed in the normal direction at the contact point. This control scheme is useful for humanoid cooperative manipulation, where is required a stable physical interaction among arms. To present this result, Section 2 introduces the nature of the dynamical problem of cooperative manipulation with redundant arms, and the proposed parametrization of the open-loop error dynamics. Section 3 describes the design procedure for the passivity-based control law. Simulation results with a humanoid robot with two redundant arms are presented in Section 4, with final discussions in Section 5 .

\section{Mathematical Modeling}

\section{$2.1 \quad$ Robot Kinematics}

Consider a robotic system composed of two redundant arms with similar kinematic structure. The joint position coordinate for each redundant arm is given by $q_{1}, q_{2} \in \Re^{n}$. In this work, we will assume that both redundant arms have the same number of degrees of freedom (DoF). Then, the forward and differential kinematics equations of each arm are given by

$$
\left.X_{i}=f_{(} q_{i}\right), \quad \dot{X}_{i}=J_{i}\left(q_{i}\right) \dot{q}_{i}
$$

with $X_{i} \in \Re^{m}$ as the end-effector pose and $J_{i}\left(q_{i}\right)=\frac{\partial f_{i}\left(q_{i}\right)}{\partial q_{i}} \in \Re^{m \times n}$ as the Jacobian matrix, for $i=1,2$. Since both arms are redundant there is not unique solution for the inverse kinematics problem because $n>m$, consequently the non-square Jacobian matrix can not be inverted. This apparent complexity turns into an attribute if we encode two different tasks within a single joint velocity desired vector $\dot{q}_{d i} \in \Re^{n}$ 1 . The former is the usual tracking task of the end-effector while the latter may be a reference to reconfigure the kinematic chain [3]:

$$
\dot{q}_{d i}=\dot{q}_{P i}+\dot{q}_{K i}
$$

Then the challenge is to design $\dot{q}_{P i}$ and $\dot{q}_{K i}$ to encode the end-effector desired velocity $\dot{X}_{d i}$ and the kinematic reconfiguration to satisfy a given cost function, respectively 2. A common approach is to use the Jacobian right pseudo-inverse $J_{i}^{+}\left(q_{i}\right)=J_{i}^{T}\left(q_{i}\right)\left[J_{i}\left(q_{i}\right) J_{i}^{T}\left(q_{i}\right)\right]^{-1} \in \Re^{n \times m}$ and its orthogonal projection matrix $I-J_{i}^{+}\left(q_{i}\right) J_{i}\left(q_{i}\right) \in \Re^{n \times n}$ to span the Jacobian kernel. Then, we define $\dot{q}_{P i}=J_{i}^{+}\left(q_{i}\right) \dot{X}_{d_{i}}$ and $\dot{q}_{K i}=\left[I-J_{i}^{+}\left(q_{i}\right) J_{i}\left(q_{i}\right)\right] \frac{\partial \Omega_{i}\left(q_{i}\right)}{\partial q_{i}}$, where $\Omega_{i}\left(q_{i}\right) \in \Re$ is a cost function to be locally optimized. The desired joint position can be computed by integrating the desired velocity $q_{d i}=\int_{t_{0}}^{t} \dot{q}_{d i} \mathrm{~d} t+q_{i}\left(t_{0}\right)$.

\footnotetext{
${ }^{1}$ Along this work, subindex $d$ and $r$ are used to denote the desired and reference values of a given variable, respectively.

${ }^{2}$ Notice that these vectors satisfy the following $\dot{q}_{P i}^{T} \dot{q}_{K i}=0$, i.e. they are orthogonal. Therefore, $\dot{q}_{i} \rightarrow \dot{q}_{d i}$ implies the achievement of both $\dot{q}_{P i}$ and $\dot{q}_{K i}$.
} 


\subsection{Holonomic Cooperation}

In order to independently control the object motion and the interaction forces, a meaningful mathematical description of the mobile holonomic constraint (object) must be synthesized. To this end, consider the following expression of the holonomic constraint $\varphi\left(X_{1}, X_{2}\right) \in \Re$ imposed by the object over the cooperative system

$$
\varphi\left(X_{1}, X_{2}\right)=\varphi_{1}\left(X_{1}\right)+\varphi_{2}\left(X_{2}\right)=0
$$

Notice that (3) means that the robotic system is in contact with a rigid object. Then, both arms must satisfy (3) when performing the trajectory. It is clear that the independent forward kinematics of each arm $X_{1}$ and $X_{2}$ can be arranged such that the scalar equation representing the holonomic constraint satisfies $\varphi_{1}\left(X_{1}\right)+\varphi_{2}\left(X_{2}\right)=0$, where $\varphi_{1}\left(X_{1}\right) \in \Re$ and $\varphi_{2}\left(X_{2}\right) \in \Re$ are scalar functions of each arm, dependant only in the corresponding end-effector's pose.

Our controller is based on the orthogonal decomposition of the contact mechanics between the object and the arms [2]. To this end, consider the following definition of the normal subspace span horizontal vector $J_{\varphi i}\left(q_{i}\right) \in \Re^{1 \times n}$ :

$$
J_{\varphi i}\left(q_{i}\right)=\frac{\nabla \varphi_{i}\left(X_{i}\right)}{\left\|\nabla \varphi_{i}\left(X_{i}\right)\right\|} J_{i}\left(q_{i}\right)
$$

where $\nabla \varphi_{i}\left(X_{i}\right)=\frac{\partial \varphi_{i}\left(X_{i}\right)}{\partial X_{i}} \in \Re^{1 \times m}$ stands for the gradient of $\varphi_{i}\left(X_{i}\right)$. The term $\frac{\nabla \varphi_{i}\left(X_{i}\right)}{\left\|\nabla \varphi_{i}\left(X_{i}\right)\right\|}$ is a unit operational vector which points out at the contact normal direction. Consider the following definition of the orthogonal projection matrix $Q_{\varphi i}\left(q_{i}\right) \in \Re^{n \times n}$ which spans the tangent subspace at the contact point:

$$
Q_{\varphi i}\left(q_{i}\right)=I-J_{\varphi i}^{+}\left(q_{i}\right) J_{\varphi i}\left(q_{i}\right)
$$

where $I \in \Re^{n \times n}$ is an identity matrix and $J_{\varphi i}^{+}\left(q_{i}\right)=J_{\varphi i}^{T}\left(q_{i}\right)\left[J_{\varphi i}\left(q_{i}\right) J_{\varphi i}^{T}\left(q_{i}\right)\right]^{-1}=$ $\frac{J_{\varphi i}^{T}\left(q_{i}\right)}{\left\|J_{\varphi i}\left(q_{i}\right)\right\|^{2}} \in \Re^{n}$ denotes the right pseudo-inverse of $J_{\varphi i}\left(q_{i}\right)$ which always exists

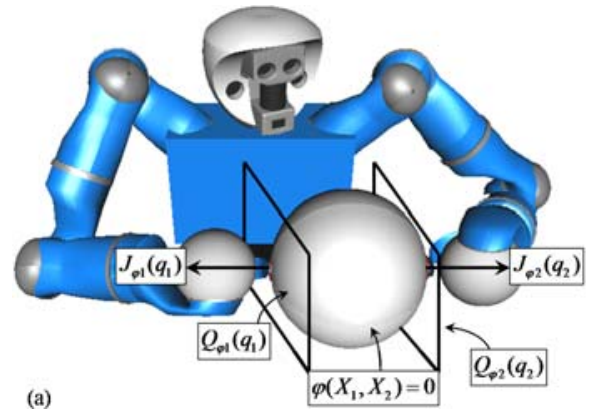

(a)

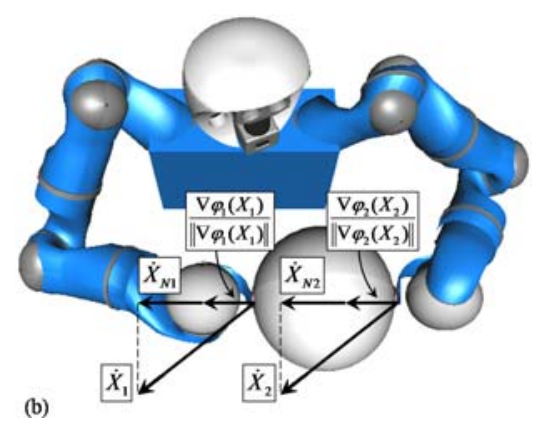

(b)

Fig. 1. (a) The vector $J_{\varphi i}\left(q_{i}\right)$ and the matrix $Q_{\varphi i}\left(q_{i}\right)$ span the normal and tangent subspaces at the contact point, respectively. (b) Conceptual representation of the endeffector constrained velocity $\dot{X}_{N i}$, which points onto the normal direction given by the unit vector $\frac{\nabla \varphi_{i}\left(X_{i}\right)}{\left\|\nabla \varphi_{i}\left(X_{i}\right)\right\|}$. 
since $J_{\varphi i}\left(q_{i}\right) J_{\varphi i}^{T}\left(q_{i}\right)=\left\|J_{\varphi i}\left(q_{i}\right)\right\|^{2} \neq 0, \forall q_{i} \in \Re^{n}$. It is evident that $Q_{\varphi i}\left(q_{i}\right)+$ $J_{\varphi i}^{+}\left(q_{i}\right) J_{\varphi i}\left(q_{i}\right)=I$, this way the normal and tangent projections of $\dot{q}_{i}$ are:

$$
\dot{q}_{i}=Q_{\varphi i}\left(q_{i}\right) \dot{q}_{i}+J_{\varphi i}^{+}\left(q_{i}\right) \dot{X}_{N i}
$$

where the scalar $\dot{X}_{N i}=J_{\varphi i} \dot{q}_{i}=\frac{\nabla \varphi_{i}\left(X_{i}\right)}{\left\|\nabla \varphi_{i}\left(X_{i}\right)\right\|} \dot{X}_{i} \in \Re$ stands for the constrained velocity [4] which is the normal component of the total operational velocity $\dot{X}_{i}$ (see Fig. 10). Notice that both arms satisfy $\dot{X}_{N 1}+\dot{X}_{N 2}=0$ when manipulating the object. This result can be easily proved by taking the time derivative of (3) as follows $\frac{d}{d t} \varphi\left(X_{1}, X_{2}\right)=\nabla \varphi_{1}\left(X_{1}\right) \dot{X}_{1}+\nabla \varphi_{2}\left(X_{2}\right) \dot{X}_{2}=0 \rightarrow \dot{X}_{N 1}+\dot{X}_{N 2}=0$.

\subsection{Cooperativeness Error}

It can be said that the cooperative arms form a closed kinematic chain which exhibits a passive joint (i.e. a non actuated joint) at the contact point. Then, a certain degree of coordination must be enforced to ensure the object manipulation while exerting a given contact force, both at the same normal direction, exactly at the two both passive joints. Notice that the traditional hybrid forceposition control [5] for holonomic constraints independently controls the force and position errors by projecting them into a normal and tangent subspace, respectively. However, this is valid only when the constraint is fixed not when the constraint is moving, such as an object being manipulated, because of the principle of virtual work. Therefore, in this paper we introduce the notion of cooperativeness error $S_{x i}=\dot{X}_{N i}-\dot{X}_{N r i} \in \Re$ that arises as a velocity-position error manifold mapped into the normal direction and whose convergence denotes the control of the normal trajectories. The constrained velocity reference value can be computed by:

$$
\dot{X}_{N r i}=\frac{\nabla \varphi_{i}\left(X_{i}\right)}{\left\|\nabla \varphi_{i}\left(X_{i}\right)\right\|}\left[\dot{X}_{d i}-\left(X_{i}-X_{d i}\right)\right]
$$

where $X_{d i}, \dot{X}_{d i} \in \Re^{m}$ are the desired end-effector trajectory. The cooperativeness error $S_{x i}$ also give us an indirect measure of the undesirable pushing-pulling effects among the robot arms, thus, the convergence of $S_{x i}$ will enforce a stable robot physical interaction.

\subsection{Robot Constrained Dynamics}

Consider a rigid and fully actuated robotic torso composed of two redundant arms 3 manipulating a rigid object. The dynamic equation is given then by the canonical Euler-Lagrange formulation as follows:

$$
\begin{aligned}
& H_{1}\left(q_{1}\right) \ddot{q}_{1}+\left[C_{1}\left(q_{1}, \dot{q}_{1}\right)+B_{1}\right] \dot{q}_{1}+g_{1}\left(q_{1}\right)=\tau_{1}+J_{\varphi 1}^{T}\left(q_{1}\right) \lambda \\
& H_{2}\left(q_{2}\right) \ddot{q}_{2}+\left[C_{2}\left(q_{2}, \dot{q}_{2}\right)+B_{2}\right] \dot{q}_{2}+g_{2}\left(q_{2}\right)=\tau_{2}+J_{\varphi 2}^{T}\left(q_{2}\right) \lambda
\end{aligned}
$$

${ }^{3}$ It is assumed that no dynamic/kinematic coupling exists among both arms, i.e., each arm is represented by an independent dynamic model. Coupling will arise through the interaction forces. 
where for the $i$-th arm, $H_{i}\left(q_{i}\right) \in R^{n \times n}$ denotes the inertia matrix, $C_{i}\left(q_{i}, \dot{q}_{i}\right) \in$ $\Re^{n \times n}$ denotes the Coriolis matrix, $B_{i} \in \Re^{n \times n}$ is the damping matrix, $g_{i}\left(q_{i}\right) \in \Re^{n}$ represents the gravity loads, and $\tau_{i} \in \Re^{n}$ stands for the joint input torques. The scalar $\lambda \in \Re$ represents the magnitude of the operational force vector $F_{i} \in$ $\Re^{m}$ exerted from the manipulated object to robot arm. Physically, the product $J_{\varphi i}^{T}\left(q_{i}\right) \lambda=J_{i}^{T}\left(q_{i}\right) \frac{\nabla \varphi_{i}^{T}\left(X_{i}\right)}{\left\|\nabla \varphi_{i}\left(X_{i}\right)\right\|} \lambda$ stands for the joint torque distribution onto the arm from the manipulated object exerted force. Notice that $\lambda$ is related with $F_{i}$ as follows $F_{i}=\frac{\nabla \varphi_{i}^{T}\left(X_{i}\right)}{\left\|\nabla \varphi_{i}\left(X_{i}\right)\right\|} \lambda$.

Now, the left hand side of (8) -(9) can be linearly parameterized in terms of a joint nominal reference $4 \dot{q}_{r i} \in \Re^{n}$ as follows [6]:

$$
H_{i}\left(q_{i}\right) \ddot{q}_{r i}+\left[C_{i}\left(q_{i}, \dot{q}_{i}\right)+B_{i}\right] \dot{q}_{r i}+g_{i}\left(q_{i}\right)=Y_{r i} \Theta_{i}
$$

where the regressor $Y_{r i}=Y_{r i}\left(q_{i}, \dot{q}_{i}, \dot{q}_{r i}, \ddot{q}_{r i}\right) \in \Re^{n \times p}$ is composed of nonlinear functions and $\Theta_{i} \in \Re^{p}$ is the vector of $p$ constant parameters. This way, the robot dynamic model (8)-(9) can be expressed as an open-loop error dynamics in terms of a new error coordinate $S_{i}=\dot{q}_{i}-\dot{q}_{r i}$. This open-loop error dynamics is useful to design the control law, because it is through the convergence of $S_{i}$ that the end-effector force and position tasks can be simultaneously achieved. To this end, by adding and subtracting $Y_{r i} \Theta_{i}$ to (8)-(9) we obtain

$$
H_{i}\left(q_{i}\right) \dot{S}_{i}+\left[C_{i}\left(q_{i}, \dot{q}_{i}\right)+B_{i}\right] S_{i}=\tau_{i}+J_{\varphi i}^{T}\left(q_{i}\right) \lambda_{i}-Y_{r i} \Theta_{i}
$$

\section{Controller Design}

\subsection{Joint Nominal Reference}

According to (6), $\dot{q}_{i}$ can be decomposed as $\dot{q}_{i}=Q_{\varphi i}\left(q_{i}\right) \dot{q}_{i}+J_{\varphi i}^{+}\left(q_{i}\right) \dot{X}_{N i}$ [4], 7]. The open-loop error coordinate is given by $S_{i}=\left[Q_{\varphi i}\left(q_{i}\right) \dot{q}_{i}+J_{\varphi i}^{+}\left(q_{i}\right) \dot{X}_{N i}\right]-\dot{q}_{r i}$. Since $\dot{q}_{r i}$ is a velocity-defined variable, it is reasonable to design this reference similarly to $\dot{q}_{i}$ to build all tracking errors, in order to preserve closed-loop passivity. Then, consider the following definition of the joint nominal reference:

$$
\dot{q}_{r i}=Q_{\varphi i}\left(q_{i}\right)\left(\dot{q}_{d i}-\alpha_{i} \Delta q_{i}\right)+J_{\varphi i}^{+}\left(q_{i}\right)\left(\dot{S}_{f}+\beta_{i} S_{f}-\dot{X}_{N j \neq i}\right)
$$

where $\Delta q_{i}=q_{i}-q_{d i} \in \Re^{n}$ is the joint position error and $S_{f}=\Delta \lambda+\int_{t_{0}}^{t} \Delta \lambda \mathrm{d} t \in \Re$ denotes the force error manifold, for $\Delta \lambda=\lambda-\lambda_{d} \in \Re$ as the contact force error. Feedback gains $\alpha_{i} \in \Re^{n \times n}$ and $\beta_{i} \in \Re$ are a positive diagonal matrix and a positive scalar, respectively. Following the same formulation as for $S_{i}$ we can say that the force error manifold is given by $S_{f}=\lambda-\lambda_{r}$, where $\lambda_{r}=\lambda_{d}-\int_{t_{0}}^{t} \Delta \lambda \mathrm{d} t \in$ $\Re$ is the force nominal reference and $\lambda_{d} \in \Re$ is the desired contact force profile. Finally, the closed-loop error coordinate is given by:

$$
S_{i}=Q_{\varphi i}\left(q_{i}\right)\left(\Delta \dot{q}_{i}+\alpha_{i} \Delta q_{i}\right)-J_{\varphi i}^{+}\left(q_{i}\right)\left(\dot{S}_{f}+\beta_{i} S_{f}\right)
$$

\footnotetext{
${ }^{4}$ Which in fact, maps the equilibrium manifold, as it becomes clear later
} 
The term $\dot{X}_{N j \neq i}$ in (12) is used to compensate in closed-loop the constrained velocity. This way, (13) is composed of two orthogonal subspaces spanned by $Q_{\varphi i}\left(q_{i}\right)$ and $J_{\varphi i}\left(q_{i}\right)$. Thus, the convergence of $S_{i}$ implies the independent convergence of position errors $\Delta \dot{q}_{i}+\alpha_{i} \Delta q_{i}$ and force errors $\dot{S}_{f}+\beta_{i} S_{f}$. On the other hand, since $\Delta \dot{q}_{i}+\alpha_{i} \Delta q_{i}$ is mapped onto the tangent subspace, its convergence can only prove position tracking along the tangent direction. Therefore, the following control law must include proper variables to ensure tracking in the normal direction, i.e., to control of the cooperativeness error $S_{x i}$.

\subsection{Control Law}

Consider the following model-based control law for the robot arm $i$ :

$$
\tau_{i}=-K_{i} S_{i}-J_{\varphi i}^{T}\left(q_{i}\right)\left(\lambda_{r}+\dot{S}_{x i}+\gamma_{i} S_{x i}\right)+Y_{r i} \Theta_{i}
$$

where $K_{i}=K_{i}^{T}>0 \in \Re^{n \times n}$ and $\gamma_{i}>0 \in \Re$ are positive feedback gains. Now, if we substitute (14) into (11), we get the following closed-loop dynamics:

$$
H_{i}\left(q_{i}\right) \dot{S}_{i}+\left[C_{i}\left(q_{i}, \dot{q}_{i}\right)+\bar{K}_{i}\right] S_{i}+J_{\varphi i}^{T}\left(q_{i}\right)\left(\dot{S}_{x i}+\gamma_{i} S_{x i}-S_{f}\right)=\tau_{i}^{*}
$$

where $\bar{K}_{i}=B_{i}+K_{i}$ and $\tau_{i}^{*}=0$ is a fictitious torque input.

Theorem. [Stable Cooperative Manipulation with Redundant Arms] Consider the robotic system (8)-(9) composed by two constrained redundant arms under the same control law (14) for each arm. The closed-loop robotic system satisfies passivity between the fictitious input torques $\tau_{1}^{*}, \tau_{2}^{*}$ and the velocity-defined variables $S_{1}, S_{2}$. Moreover, for each redundant arm, asymptotic convergence for the end-effector force $\Delta \lambda$, for the joint position $\Delta q_{i}$ and for cooperativeness $S_{x i}$ tracking errors are achieved. Additionally, a local minimum of a cost function $\Omega_{i}\left(q_{i}\right)$ is reached by the dynamic reconfiguration of each redundant arm.

Proof. Through the following passivity analysis: $P=\sum_{i=1}^{2} S_{i}^{T} \tau_{i}^{*}=\dot{V}+P_{d i s s}$, a candidate Lyapunov function $V$ is found for the closed-loop robotic system as follows:

$$
\sum_{i=1}^{2} S_{i}^{T} \tau_{i}^{*}=\sum_{i=1}^{2} \frac{\mathrm{d}}{\mathrm{d} t} \frac{1}{2}\left[S_{i}^{T} H_{i}\left(q_{i}\right) S_{i}+S_{f}^{2}+S_{x i}^{2}\right]+\sum_{i=1}^{2}\left[S_{i}^{T} \bar{K}_{i} S_{i}+\beta_{i} S_{f}^{2}+\gamma_{i} S_{x i}^{2}\right]
$$

where $V=\sum_{i=1}^{2} \frac{1}{2}\left[S_{i}^{T} H_{i}\left(q_{i}\right) S_{i}+S_{f}^{2}+S_{x i}^{2}\right] \geq 0$ qualifies as a candidate Lyapunov function. Since $\tau_{i}^{*}=0$, thus $\dot{V}=-\sum_{i=1}^{2}\left(S_{i}^{T} \bar{K}_{i} S_{i}+\beta_{i} S_{f}^{2}+\gamma_{i} S_{x i}^{2}\right) \leq 0$, proving stability. Then the positive definite feedback gains $K_{i}, \beta_{i}$ and $\gamma_{i}$ can be employed to modify the transient performance of the system. It is clear that $V(t) \leq V\left(t_{0}\right)$; also, notice that $S_{i}, S_{f}, S_{x i} \in \mathcal{L}_{\infty}$ and $S_{i}, S_{f}, S_{x i} \in \mathcal{L}_{2}, \Rightarrow S_{i}, S_{f}, S_{x i} \in \mathcal{L}_{2} \cap \mathcal{L}_{\infty}$, and by invoking the Direct Lyapunov Theorem we have that $S_{i}, S_{f}$ and $S_{x i}$ converge asymptotically into the equilibrium point, that is $S_{i}, S_{f}, S_{x i} \rightarrow 0$ as 
$t \rightarrow \infty$. Notice the explicit convergence of $S_{x i}$, this stands as the paper major contribution. Finally, to prove the local optimization of $\Omega_{i}\left(q_{i}\right)$, consider the following: The scalar cost function $\Omega_{i}\left(q_{i}\right)$ has a local minimum at point $q_{i}^{*}$, if there is a vicinity around $q_{i}^{*}$ defined by positive scalar $\epsilon_{i}>0$ such that for all points $q_{i}$ in this vicinity that satisfy $\left\|q_{i}-q_{i}^{*}\right\|<\epsilon_{i}$, the increment of $\Omega_{i}\left(q_{i}\right)$ has the same sign. If $\Omega_{i}\left(q_{i}\right)-\Omega_{i}\left(q_{i}^{*}\right) \geq 0$, then $\Omega_{i}\left(q_{i}^{*}\right)$ is a local minimum. Considering the definition of $\dot{q}_{K i}=\left[I-J_{i}^{+}\left(q_{i}\right) J_{i}\left(q_{i}\right)\right] \frac{\partial \Omega_{i}\left(q_{i}\right)}{\partial q_{i}}$, it is evident that $\dot{q}_{K i}$ moves in the direction on which $\Omega_{i}\left(q_{i}\right)$ decreases, then $\dot{q}_{K i}$ vanishes at $q_{i}^{*}$, thus $\Omega_{i}\left(q_{i}\right)$ is locally optimized. QED

\section{Simulation Study}

Settings. In order to validate the algorithm, a simulation study was carried out using the full dynamic model of a robot-torso, based on the DLR JustinC, with two identical 7 DoF arms manipulating a rigid object.

Manipulating the object. We want to cooperatively move an object along the $x$-axis, assuming that both arms are already in contact with the object. The desired object pose trajectory is given by: $X_{d}=\left[x_{d}, y_{d}, z_{d}, \phi_{d}, \theta_{d}, \psi_{d}\right]=$ $[0.1 \sin (t),-0.1,0.05,0,0,0]$, where $x_{d}, y_{d}, z_{d}(\mathrm{~m})$ are the cartesian coordinates and $\phi_{d}, \theta_{d}, \psi_{d}(\mathrm{rad} / \mathrm{seg})$ the euler angles. Simultaneously, the object must be hold with the following exerted force profile $\lambda_{d}=100+20 \tanh (0.1 t) \mathrm{N}$.

Redundancy task. Since the desired force profile is increasing with time, the robot kinematic chain is reconfigured in order to protect the weaker wrist joints. This way, redundancy is exploited to overcome the robot joints physical limitations.

Results. On Figure 2 it can be seen that due to the increase in the force profile (arrow size), each redundant arm is dynamically reconfigured in order to satisfy the force requirements. As a consequence of the reconfiguration, the exerted contact force is mainly achieved by the shoulder joints.

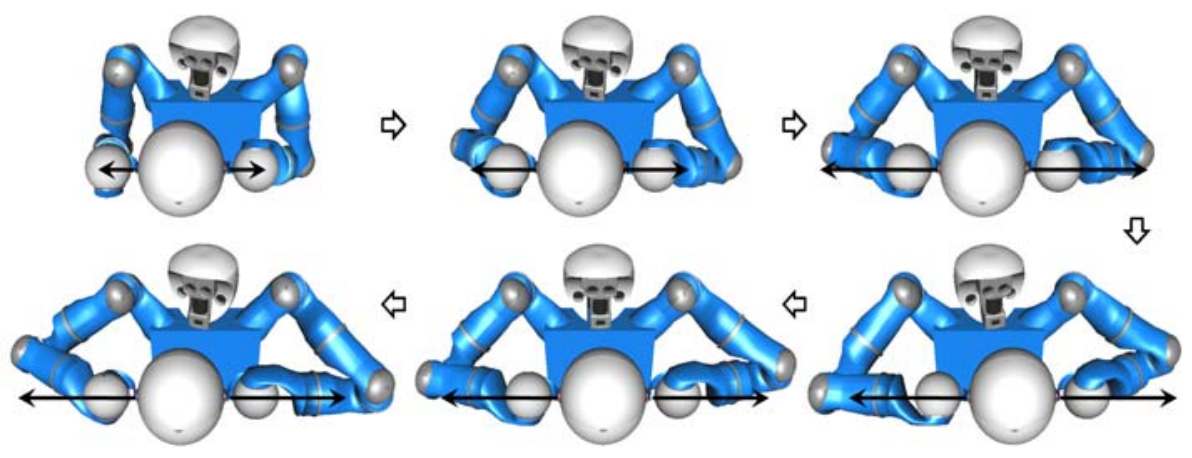

Fig. 2. Kinematic reconfiguration along the object trajectory 


\section{Conclusions}

As the major contribution, our paper proves closed-loop stability in the Lyapunov sense of the cooperativeness error $S_{x i}$. This result is useful to guarantee simultaneous force-position trajectory tracking of the manipulated object in the constrained (normal) direction, despite the exerted interaction forces. The passivity-based computed-torque like controller has been derived with strict closed-loop stability proofs. Therefore, the extension to other passivity motivated control schemes such as adaptive control, sliding-modes control, cartesian control is straightforward. Notice that since redundancy only reconfigures the kinematic structure without changing the end-effector pose, then it has not direct implication with the convergence of $S_{x i}$. Therefore, redundancy is here employed to enhance the manipulation capabilities of the system, such as avoiding obstacles, protecting weaker joints, avoiding joint limits, among others.

\section{References}

1. Zivanovic, M.D., Vukobratovic, M.K.: Multi-arm cooperating robots. Springer, Heidelberg (2006)

2. Arimoto, S., Liu, Y.H., Naniwa, T.: Model-based adaptive hybrid control for geometrically constrained robots. In: Int. conference on robotics and autom., pp. 618-623 (1993)

3. Nakamura, Y.: Adv. robotics: redundancy and optimization. Addison-Wesley, Reading (1991)

4. Liu, Y., Arimoto, S., Parra-Vega, V., Kitagaki, K.: Decentralized adaptive control of multiple manipulators in cooperations. Int. journal of control, 649-673 (1997)

5. Arimoto, S.: Joint-space orthogonalization and passivity for physical interpretations of dextrous robot motions under geometric constraints. Int. Journal of Robust and Nonlinear Control (1992)

6. Slotine, J.J., Li, W.: On the adaptive control of manipulators. The international journal of robotics research, 49-59 (1987)

7. Navarro-Alarcon, D., Parra-Vega, V., Olguin-Diaz, E.: Minimum set of feedback sensors for high performance decentralized cooperative force control of redundant manipulators. In: Int. workshop on robotic and sensors environments, pp. 114-119 (2008) 